\title{
Botanique, chimie et toxicologie des solanacées hallucinogènes : belladone, datura, jusquiame, mandragore
}

\section{Botanic, chemistry and toxicological review of the hallucinogen solanacies : deadly nightshade, jimsonweed, henbane, mandrake}

\section{Jean-Pierre GOULLÉ(1)*, Gilbert PÉPIN(2), Véronique DUMESTRE-TOULET ${ }^{(3)}$, Christian LACROIX ${ }^{(1)}$}

(1) Laboratoire de Pharmacocinétique et de Toxicologie Cliniques, Groupe Hospitalier du Havre BP 24 - 76083 LE HAVRE CEDEX

(2) Laboratoire d'Expertises TOXLAB, 7, rue Jacques Cartier - 75018 PARIS (3) Laboratoire TOXGEN, 11, rue du Commandant Cousteau - 33100 BORDEAUX

* Auteur à qui adresser la correspondance : Jean-Pierre GOULLÉ, Laboratoire de Pharmacocinétique et de Toxicologie Cliniques, Groupe Hospitalier du Havre, BP 24 - 76083 LE HAVRE CEDEX - FRANCE Tél : 0232733223 - Fax : 0232733238 -E-mail : jgoulle@ch-havre.fr

\section{$\boldsymbol{R} \boldsymbol{E} \boldsymbol{S U M E}$}

Ces quatre plantes de la famille des Solanaceae ont une histoire riche de légendes et de superstitions. Pendant plusieurs siècles, on donna à la mandragore un caractère magique, et des propriétés médicinales, mais elle n'est plus utilisée aujourd'hui. En revanche les trois autres plantes le sont encore pour leurs vertus médicinales, surtout en homéopathie. En médecine allopathique, outre l'atropine et son oxyde, on trouve des spécialités contenant de la scopolamine mais également de la poudre de belladone, ou de l'hyoscyamine. Après une description botanique de la belladone, de la jusquiame, du datura, et de la mandragore, les auteurs précisent la nature et la structure des alcaloïdes contenus dans ces végétaux : l'hyoscyamine, la scopolamine et l'atropine. Chez l'homme, ces alcaloüdes exercent une action parasympatholytique pouvant conduire en cas d'intoxication à un syndrome atropinique ou anticholinergique. Celui-ci est caractérisé par un blocage de la production d'acétylcholine

\section{SUMMARY}

These four Solanaceae have a rich history of legends and superstitions, particularly mandrake. For many centuries this plant was considered magic, with medical properties, but is no longer used. On the other hand the three others are still used for their medical properties, particularly in the homoeopathic medications. Atropine and its oxide, scopolamine, hyoscyamine, deadly nightshade powder, are found in patent allotropic medicines. After a botanical description of deadly nightshade, jimsonweed henbane and mandrake, the authors study the nature and the structure of the alkaloids found in these vegetals : hyoscyamine, scopolamine and atropine. These alkaloids exert a parasympatholytic action in man, which may lead to atropinic i.e. anticholinergic syndrome. This syndrome is characterized by acetylcholine blockage in the central and peripheric nervous systems synapses. Typical anticholinergic syndrome includes confusion, agitation, hallucinations, combative behaviour, myo- 
dans les synapses du système nerveux central et périphérique. Les principaux signes cliniques associés au blocage périphérique sont mydriase, sécheresse cutanée et muqueuse, vasodilatation et tachycardie. Le blocage central est responsable de confusion, d'agitation, d'hallucinations, de myoclonies, de convulsions, de coma et de dépression respiratoire. Lors de certaines intoxications, d'authentiques syndromes atropiniques peuvent être constatés. Bien que rares, ils peuvent cependant être mortels malgré le traitement symptomatique et la réanimation. Des observations d'intoxications sont présentées. Parmi les 150000 intoxications signalées chaque année aux centres antipoison français, les végétaux représentent moins de $3 \%$ des cas, mais dans l'enquête sur la consommation de drogues réalisée en 2002 chez les jeunes gens de 17 à 19 ans par l'observatoire français des drogues et des toxicomanies, le datura est cité en sixième position parmi les principales autres drogues. L'analyse toxicologique de ces composés est extrêmement délicate en raison des faibles concentrations susceptibles d'exercer une action pharmacologique. Celle-ci fait principalement appel à la chromatographie en phase liquide couplée à la détection de masse (CL-SM), et à la détection de masse en mode tan$\operatorname{dem}(C L H P-S M / S M)$.

\section{MOTS-CLÉS}

Atropine, scopolamine, hyoscyamine, hallucinogène.

\section{Introduction}

La belladone, le datura, la jusquiame et la mandragore appartiennent à l'importante famille végétale des solanacées qui compte environ 2000 espèces, dont les trois quarts sont inclus dans le genre solanum. Plusieurs représentants de cette famille figurent parmi les végétaux les plus consommés : pomme de terre, tomate, aubergine, et tabac en particulier. On les rencontre aussi bien dans les zones tropicales que tempérées, avec de nombreuses espèces originaires d'Amérique du sud et importées dans nos régions. La toxicité des premières espèces présentes naturellement en Europe a laissé une mauvaise réputation aux plantes importées d'Amérique comme la tomate ou la pomme de terre. Belladone, datura, jusquiame et mandragore ont une histoire riche de légendes et de superstitions ; utilisées pour leurs propriétés médicinales depuis des millénaires, allant même jusqu'à donner un caractère magique à la mandragore. A l'exception de cette dernière, les trois autres plantes sont encore utilisées de nos jours pour leurs vertus médicinales surtout en homéopathie. En médecine allopathique, outre l'atropine et son oxyde, on trouve des spécialités contenant de la scopolamine, mais également de la poudre de belladone, ou de l'hyoscyamine. En dehors de l'usage médical qui peut être fait de ces végétaux des cas d'intoxication sont rapportés. Il s'agit par exemple d'intoxications accidentelles consécutives à l'ingestion de fruits noirs de belladone confondus avec des cerises, ou plus clonies, convulsions coma and pulmonary failure. Real atropinic syndromes are observed during intoxications but they stay rare. They may lead to death despite symptomatic and intensive care treatment. Some real cases are presented. Plant poisoning is not frequent, it is less than $3 \%$ of the 150 000 intoxications collected by French poison centers. Nevertheless, inquiry among young people 17-19 year-old about drug abuse revealed that datura is one of the most used among the other main drugs (rank 6). Alkaloids toxicological analysis is very difficult due to very low concentrations that may lead to pharmacological effects. Screening and quantitation methods to confirm the nature of the intoxication are gas chromatography and liquid chromatography coupled with mass spectrometry and mass tandem spectrometry.

\section{KEY-WORDS}

Atropine, scopolamine, hyoscyamine, hallucinogen.

récemment d'une augmentation des intoxications volontaires liées à la consommation de datura pour ses propriétés hallucinogènes. Si parmi les 150000 intoxications signalées chaque année aux centres antipoison français, les végétaux ne représentent que moins de $3 \%$ des cas (1). Dans l'enquête réalisée en 2002 par l'observatoire français des drogues et des toxicomanies sur la consommation de substances psychoactives chez les jeunes français de 17 à 19 ans, le datura arrive en sixième position ( $3 \%$ des citations), après la morphine, mais avant l'ecstasy, la kétamine ou le subutex® (2). Il nous a de ce fait semblé indispensable dans ce numéro spécial plantes et champignons hallucinogènes, de consacrer un article aux solanacées.

\section{Botanique $(3,4)$}

La famille des solanacées appartient au deuxième groupe végétal des tubiflores qui comporte 6 familles de 2000 espèces environ chacune. Elles présentent quelques caractères communs : ce sont essentiellement des herbes, bien représentées dans les zones tempérées et froides, l'ovaire présente un caractère peu évolué, apparition de la zygomorphie, l'androcée est réduit souvent à quatre étamines, le feuillage est plutôt alterne. La famille des solanacées est regroupée autour du genre solanum. Linné nommait les plantes de cette famille les «blêmes», les «tristes» car les feuilles ont plutôt un aspect tombant avec des couleurs assez ternes. 


\section{- La belladone (Atropa belladonna): (photographies 1 et 2)}

L'origine du nom latin, Atropa belladonna, vient d'Atropos la déesse de la mort et de belladonna qui signifie belle dame car le suc des baies était utilisé comme fard par instillation dans les yeux pour agrandir le regard et le rendre plus éclatant en raison de la dilatation des pupilles qui s'ensuivait. Elle porte parfois pour nom commun, l'herbe empoisonnée, la belle dame, ou la morelle furieuse. La belladone est une plante vivace de 50 à $150 \mathrm{~cm}$, très ramifiée et d'odeur fétide, lorsqu'elle est froissée, due à la présence de poils sécréteurs. Elle présente des feuilles alternes ou opposées à court pétiole. Ses fleurs qui mesurent 2 à $3 \mathrm{~cm}$, sont en forme de cloche à 5 dents violettes, brunes ou verdâtres. Elles donnent des baies globuleuses de 1 à $1,5 \mathrm{~cm}$, noires, brillantes, entourées d'un calice à 5 sépales. La floraison s'étale de juin à août. Elle pousse de manière disséminée de préférence dans les haies, les clairières en sol calcaire, jusqu'à une altitude de $1500 \mathrm{~m}$. On récolte les feuilles en été et les racines à partir de la première année en automne. Les organes les plus riches en alcaloïdes sont les racines, mais toutes les parties de la plante sont toxiques à des degrés divers.

- Les jusquiames (Hyoscyamus niger, Hyoscyamus albus) : (Photographie 3)

Ce genre comprend 15 espèces annuelles, bisannuelles ou vivaces, vivant sur les talus, les falaises, les friches et les plages de galets en Europe, en Afrique du nord et en Asie.

La jusquiame noire (Hyocyamus niger) :

Il s'agit d'une plante commune dans toute l'Europe tempérée. Elle porte pour nom commun l'herbe aux engelures, la mort aux poules, l'herbe des teigneux. Elle est annuelle ou bisannuelle, collante, à odeur forte désagréable, et mesure de 0,60 à $1,20 \mathrm{~m}$ de hauteur. Les feuilles, de 10 à $20 \mathrm{~cm}$ de long sont ovales; lancéolées, entières ou dentées et recouvertes d'un épais feutrage gris vert et groupées en rosettes basales. De juin à octobre, les fleurs qui mesurent de 1 à $3 \mathrm{~cm}$ de diamètre ont 5 lobes, elles sont de couleur jaune terne, avec le centre et des veines pourpres, elles sont solitaires ou réunies par paires à l'extrémité de grappes arquées, ramifiées. Les fruits, s'ouvrant à maturité par une sorte d'opercule circulaire, sont secs et appréciés dans les compositions florales. La racine est pivotante, velue, collante et dégage également une odeur forte.

\section{La jusquiame blanche (Hyoscyamus albus) :}

Cette variété également annuelle ou bisannuelle, qui mesure de 30 à $90 \mathrm{~cm}$ de hauteur, à port dressé, a des feuilles plus petites que la jusquiame noire $(5$ à $10 \mathrm{~cm}$ de long), elles sont larges, ovales, collantes et de couleur vert clair. Au printemps, la floraison donne des fleurs de 1 à 3 $\mathrm{cm}$ de long, bilabiées, irrégulièrement lobées, de couleur vert pâle.

- Le genre datura (D. stramonium, D. innoxia, D. metel,
D. ferox, D. ceratoacula, D. fastuosa, D. quercifolia, $D$. wrightii) et brugmansia (B. arborea, B. aurea, B. sanguinea, $B$. suaveolens, $B$. versicolor, $B$. vulcanicola) : (photographies 4 et 5)

Datura stramonium est la variété la plus connue sous nos latitudes, il en existe d'autres que l'on rencontre essentiellement dans les pays tropicaux.

\section{Le datura stramoine (Datura stramonium) :}

C'est une plante herbacée annuelle de $50 \mathrm{~cm}$ à $1 \mathrm{~m}$ de haut, originaire d'Orient, qui croît communément en France dans les décombres et les lieux incultes. On lui donne parfois le nom de pomme épineuse. Les feuilles sont grandes et dentées. Les fleurs en forme de trompette ont une couleur blanche, elles donnent naissance au fuit non charnu qui est une capsule verte, ovale chargée d'aiguillons robustes. Ce dernier renferme des graines noires d'apparence agréable et particulièrement riches en alcaloïdes.

\section{Le brugmansia en arbre appelé à tort le datura en arbre (Brugmansia arborea ou Datura arborea) :}

Appelé communément, par erreur, stramoine en arbre ou trompette des anges, il s'agit en réalité d'un genre distinct du genre datura comme l'ont montré des études récentes sur la biologie de ces plantes. C'est un arbuste, ou un arbre de 2 à $3 \mathrm{~m}$ pouvant atteindre $5 \mathrm{~m}$ de haut originaire d'Amérique du sud. En France, il peut être cultivé en serre dans le nord, ou en plein air en provence. Il est constitué de grosses tiges charnues et fragiles, pourvues de larges feuilles velues et molles. Les fleurs sont énormes, pendantes, d'un blanc pur, dont les cornets innombrables sont d'un effet superbe au moment de la floraison qui peut se produire deux ou trois fois dans l'année. Les fleurs qui ne durent qu'un jour sont immédiatement remplacées par une autre, si bien que la floraison donne l'impression d'être continue ; elles répandent un parfum suave. Le fruit contient un grand nombre de graines.

Autres daturas et brugmansias :

Il existe de nombreuses autres espèces de datura. Le nom de brugmansia doit être définitivement réservé aux arbustes à fleurs pendantes et celui de datura aux herbacées vivaces ou annuelles à fleurs dressées ou horizontales.

- Les mandragores (M. officinarum, M. automnalis, $M$. caulescens, M. vernalis) : (Photographie 6)

Les mandragores sont des plantes herbacées des pays du pourtour méditerranéen. Elles sont " décevantes », sans tiges, avec de grandes feuilles molles près du sol comme la laitue. Les fleurs d'automne blanches ou mauves, donnent naissance à des baies jaunes ou rouges qui réémettent la lumière blanche. La racine est une rave impressionnante, d'une taille pouvant atteindre 60 à $80 \mathrm{~cm}$; elle peut peser plusieurs kilos et présente une vague apparence humaine avec un tronc, des jambes. 


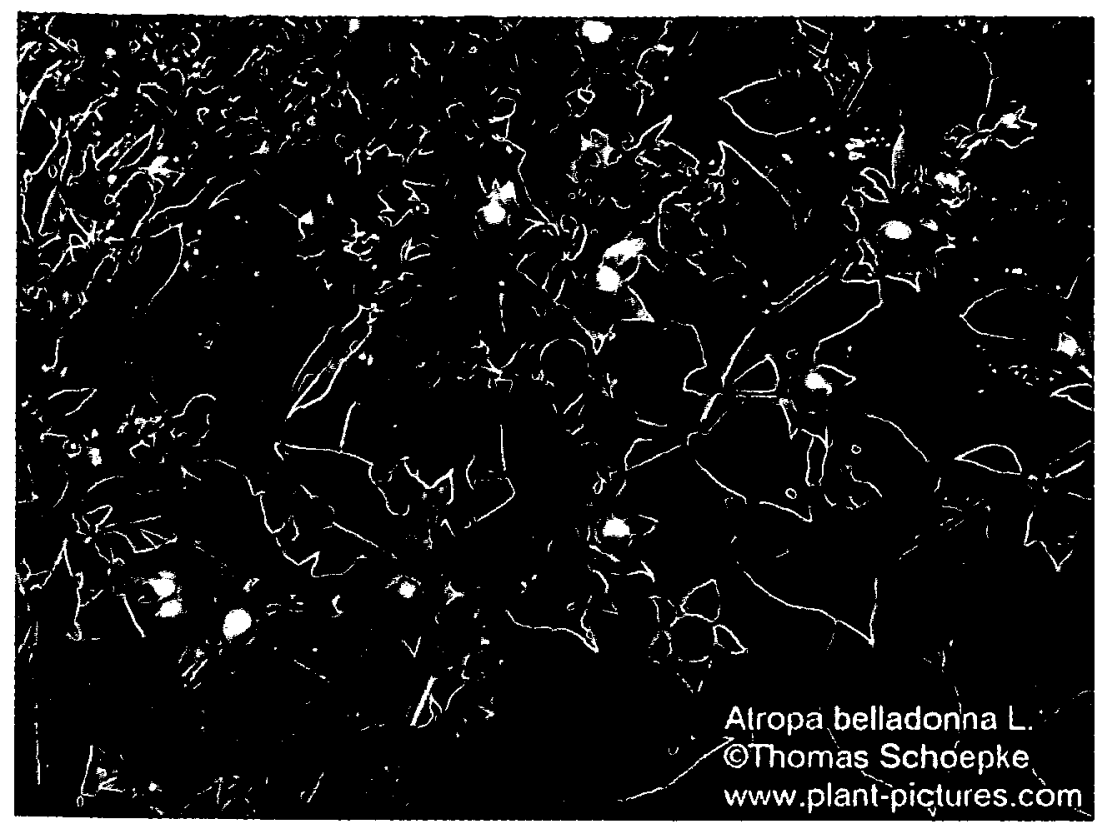

Photo 1 : Belladone photo Thomas SCHOEPKE: http://www.pharmakobotanik.de/gallery/gal-sola.htm

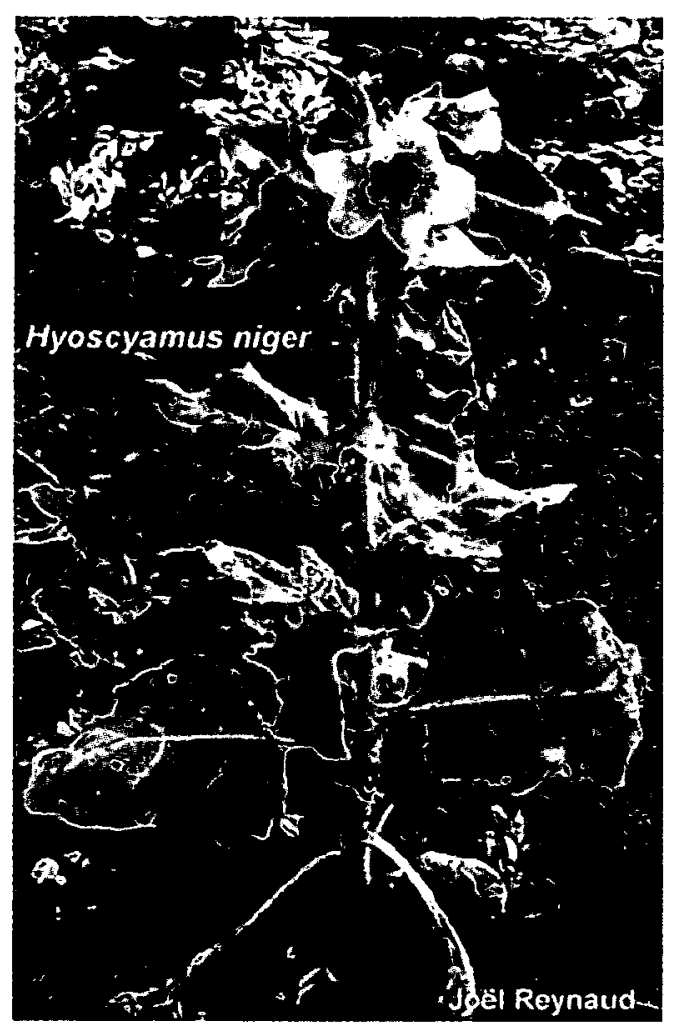

Photo 3 : Jusquiame photo Joël REYNAUD : http://ispb.univ-lyonl.fr/cours/botaniquel

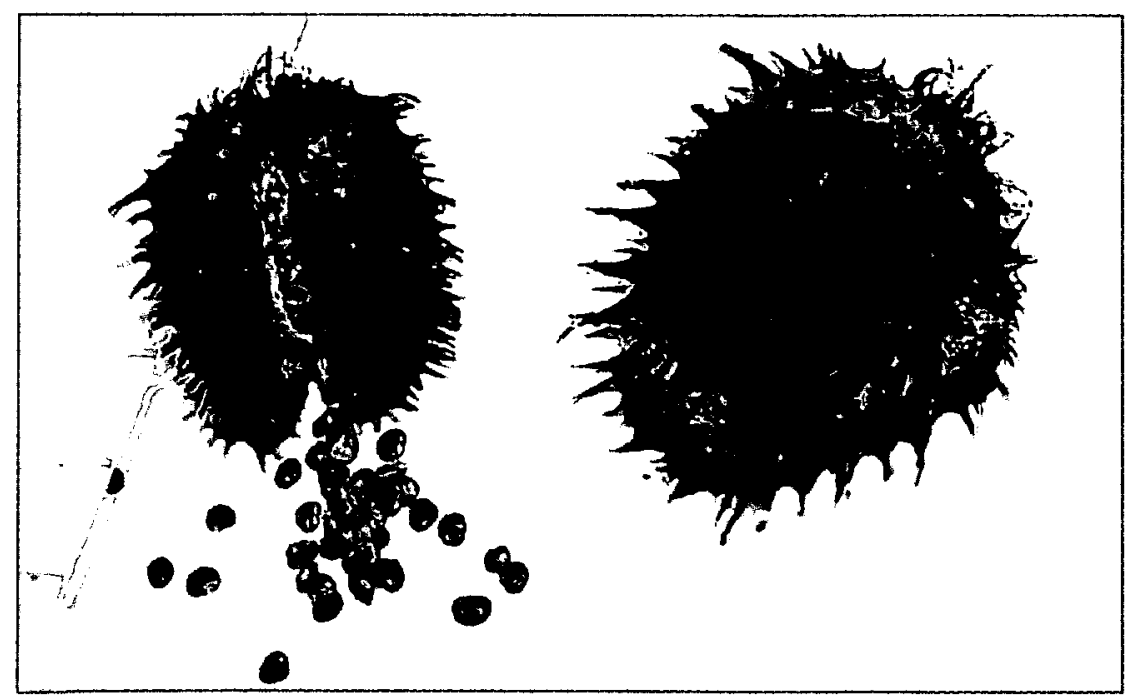

Photo 5 : Datura photo Véronique DUMESTRE-TOULET

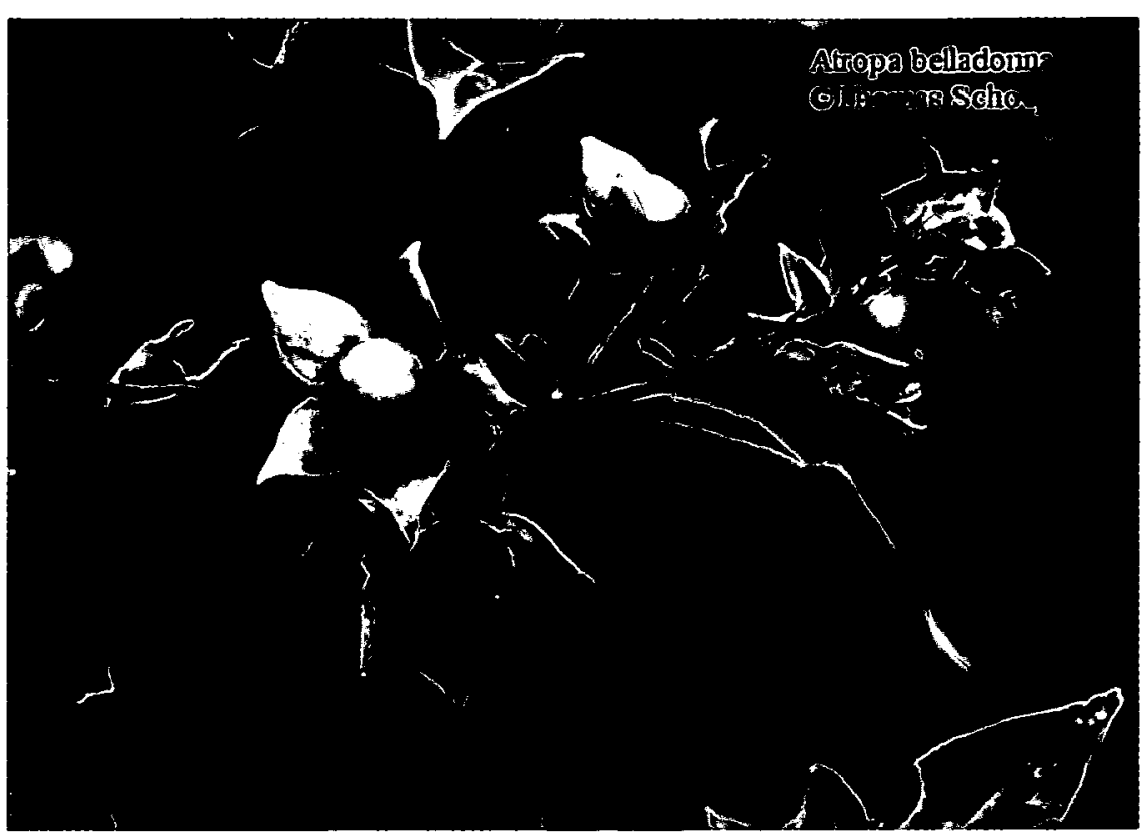

Photo 2 : Belladone photo Thomas SCHOEPKE: http://www.pharmakobotanik.de/gallery/gal-sola.htm

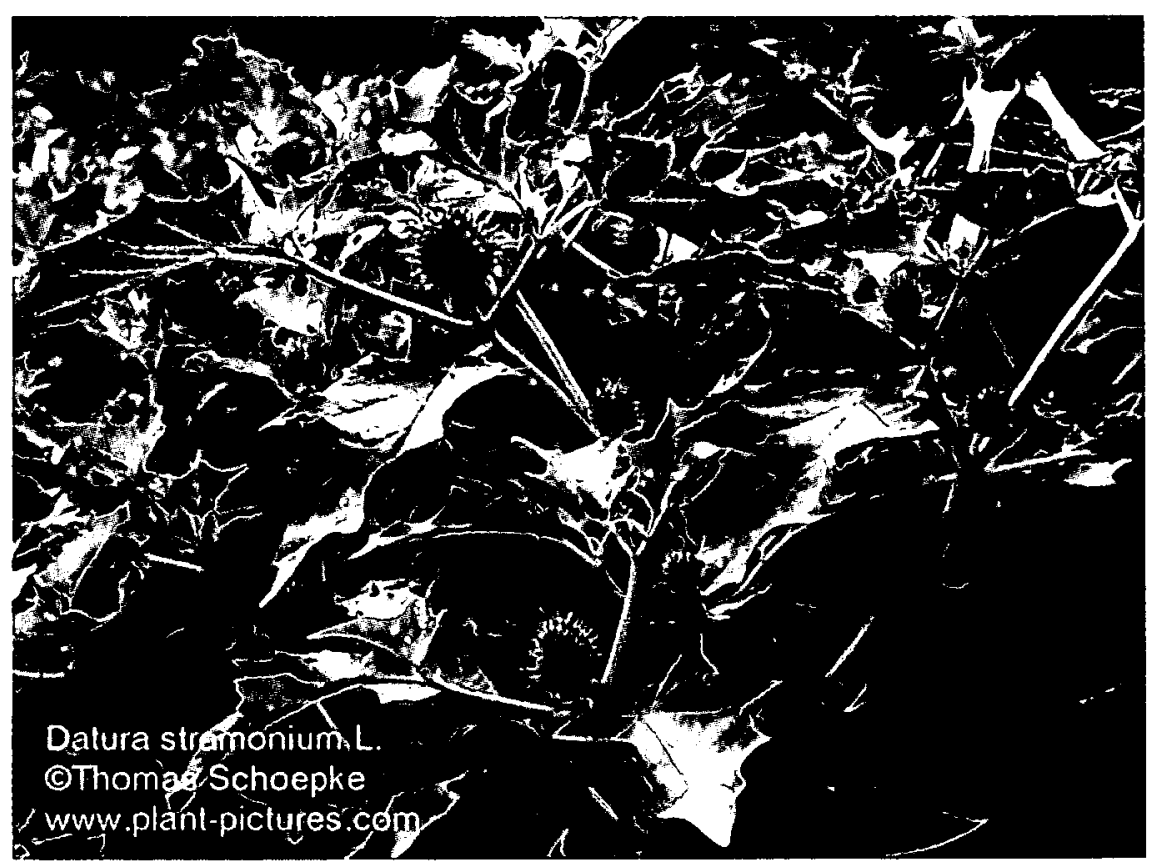

Photo 4 : Datura photo Thomas SCHOEPKE : http://www.pharmakobotanik.de/gallery/gal-sola.htm

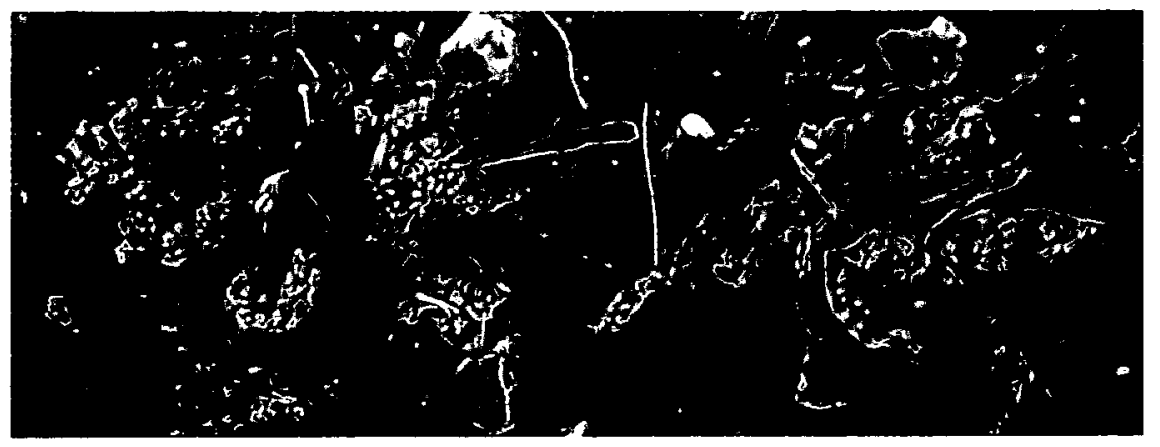

Photo 6 : Mandragore photo Albert PERDECK : http://www.csdl.tamu.edu/FLORA 


\section{Principes actifs (5-9)}

\section{- Structure :}

Les solanacées dont nous venons de faire la description botanique contiennent outre de nombreux principes actifs végétaux, des alcaloïdes dérivés du tropanol et de la scopoline (figure 1). Atropine et daturine sont des esters tropiques du tropanol, alors que la scopolamine ou hyoscine est un ester tropique de la scopoline. L'hyoscyamine (daturine) de formule brute $\mathrm{C}_{17} \mathrm{H}_{23} \mathrm{NO}_{3}$, qui est une base lévogyre se transforme facilement en DL-hyoscyamine (atropine) forme racémique dont la formule brute est identique. C'est une racémisation qui s'explique facilement, car l'atropine doit être considérée comme l'ester résultant de l'action de l'acide DLtropique sur le tropanol, tandis que l'hyoscyamine est le produit de l'estérification du tropanol par l'acide Ltropique. L'isomérie entre l'atropine et l'hyoscyamine est donc le fait de l'acide tropique qui, possédant un carbone asymétrique existe sous les trois formes isomères : droite, gauche et racémique. La scopolamine $\left(\mathrm{C}_{17} \mathrm{H}_{21} \mathrm{NO}_{4}\right)$ dont la structure est très voisine de l'atropine et de l'hyoscyamine possède en plus de ces deux molécules un atome d'oxygène sur le noyau tropane. Ces alcaloïdes donnent naissance à de nombreux autres composés dont l'atropamine ou apoatropine, et la belladonine, formes isomères, ne différant de l'atropine et de l'hyoscyamine que par départ d'une molécule d'eau. Ces dérivés conduisent par hydrolyse à l'acide atropique ainsi que respectivement au tropanol et à la scopoline. Il convient de mentionner, que dans l'histoire chimique de ces alcaloïdes, un point important, est la propriété qu'ils possèdent de se transformer assez facilement les uns dans les autres.

\section{- Teneurs :}

Pour la belladone, les organes les plus actifs sont les racines, mais toutes les parties de la plante contiennent des principes actifs à des concentrations diverses. La proportion d'alcaloïdes totaux varie, dans la feuille, selon l'époque à laquelle on l'a récoltée et selon que la plante est sauvage ou cultivée, cette dernière est plus riche en alcaloïdes. La concentration en alcaloïdes est comprise entre $0,3 \%$ et $1 \%$ de plante sèche; atropine et L-hyoscyamine représentent 90 à $95 \%$ des alcaloïdes totaux et la scopolamine 5 à $10 \%$. Il existe en outre certains alcaloïdes mineurs dérivés du tropane que sont la tigloyltropéine, l'aposcopolamine, l'apoatropine, l'hydroxy-hyoscyamine et le tigloyoxytropane, ainsi qu'un alcaloïde pyrrolidinique : l'hygrine. Les feuilles de belladone renferment aussi une petite quan-

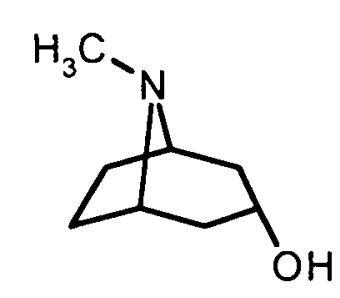

tropanol

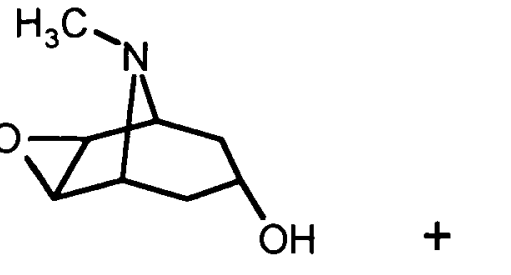

scopoline acide tropique

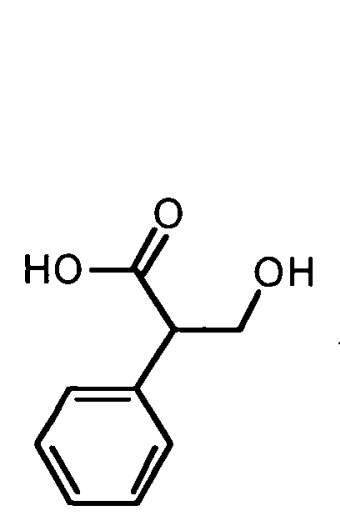

acide L-tropique

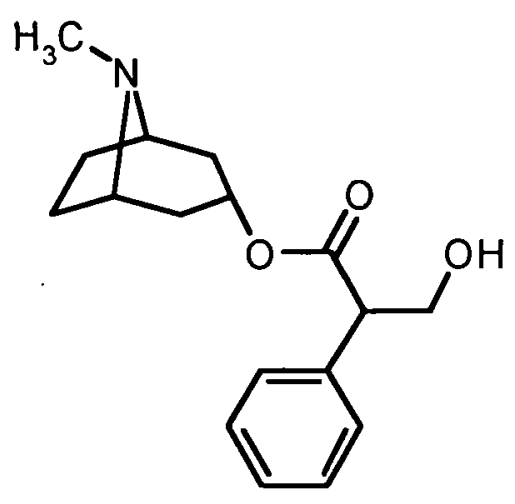

$D, L$-atropine (racémique)

L-hyoscyamine (lévogyre)
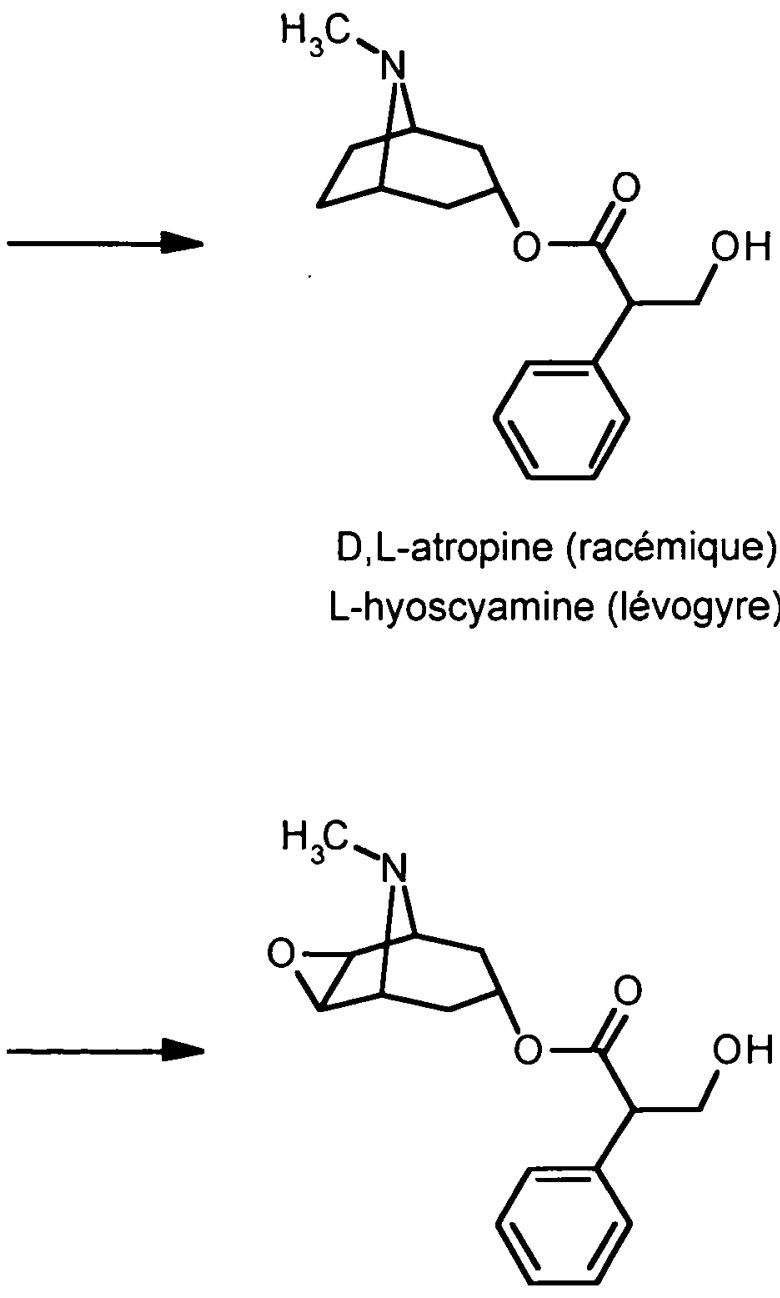

scopolamine

Figure 1 : Synthèse de l'atropine, de l'hyoscyamine et de la scopolamine à partir de l'acide tropique. 
tité d'une coumarine, le scopolétol. La jusquiame, moins toxique que la belladone, contient de 0,2 à $0,5 \%$ d'alcaloïdes, surtout sous forme de L-hyoscyamine mais également sous forme d'atropine et de scopolamine. Dans les datura, la teneur en alcaloïdes oscille entre 0,2 et $0,6 \%$, mais contrairement à la belladone et à la jusquiame contenant presque exclusivement de la Lhyoscyamine et de l'atropine, ces deux molécules ne représentent que $67 \%$ des alcaloïdes totaux de la plante sèche, alors que la scopolamine atteint $33 \%$. Les fleurs séchées de datura metel appelées yangjinhua contiennent $85 \%$ de scopolamine et $15 \%$ de L-hyoscyamine et d'atropine. Cette variété de datura renferme également un alcaloïde secondaire caractéristique : la météloïdine. La chimie des brugmansia montre que ces solanacées contiennent les mêmes alcaloïdes que les datura : scopolamine, L-hyoscyamine, atropine et divers alcaloïdes secondaires du groupe tropane comme la norscopolamine, l'aposcopolamine, la météloïdine. La scopolamine est présente en grande quantité. Les feuilles et les tiges de Brugmansia aurea par exemple contiennent $0,3 \%$ d'alcaloïdes dont $80 \%$ de scopolamine. On en trouve également dans les racines. En ce qui concerne les mandragores, la scopolamine est l'alcaloïde essentiel, mais elles contiennent également en moindre quantité de la L-hyoscyamine et de l'atropine.

\section{- Relation structure-activité :}

\section{L-hyoscyamine et atropine :}

Ces molécules sont les chefs de file d'une série de substances susceptibles d'agir comme antagonistes des récepteurs muscariniques. Les antagonistes des récepteurs muscariniques s'opposent par un blocage compétitif et réversible des récepteurs périphériques et centraux à l'action de l'acétylcholine. En règle générale, ces antagonistes des récepteurs muscariniques ont une faible activité antagoniste sur les effets de l'acétylcholine sur les récepteurs nicotiniques. Des études autoradiographiques ont révélé une large distribution des récepteurs muscariniques dans le cerveau humain. On admet que l'essentiel des effets exercés par des doses thérapeutiques d'atropiniques sur le système nerveux central, sont vraisemblablement liés à une inhibition centrale muscarinique. Seules les formes lévogyres (Lhyoscyamine, fraction $\mathrm{L}$ de la DL-atropine) ont une activité sur les récepteurs muscariniques. Pour que l'action pharmacologique puisse s'exercer sur les récepteurs muscariniques, l'ester de la tropine et de l'acide tropique doivent être intacts. En effet, l'acide tropique seul, le tropanol ou la scopoline seuls ne montrent aucune activité antimuscarinique significative. La présence d'un radical $\mathrm{OH}$ libre sur la portion acide de l'ester est également importante pour l'activité pharmacologique.

\section{Scopolamine :}

La scopolamine ou L-hyoscine montre une activité comparable à la L-hyoscyamine et à l'atropine en raison d'une structure très voisine. Toutefois, la présence d'un pont oxygène dans le noyau tropane lui confère des propriétés particulières du fait d'un meilleur passage à travers la barrière hémato-encéphalique.

\section{Modes de consommation, ethnologie $(7,10)$}

Ces quatre plantes de la famille des solanacées ont une histoire extrêmement riche, leur consommation remontant à plusieurs millénaires. Outre des buts thérapeutiques, la consommation de drogues psychoactives, sans doute aussi ancienne que l'espèce humaine, a servi principalement des objectifs magico-religieux et récréatifs. Aujourd'hui, la consommation de substances psychoactives reste associée dans quelques sociétés traditionnelles à des rituels chamaniques, notamment en Sibérie et chez les Indiens d'Amérique, ou religieux, en particulier chez les hindous. Les plantes qui sont utilisées dans ce but ont un caractère sacré. Depuis trois décennies, l'usage profane de drogues, essentiellement à des fins récréatives s'est répandu dans le monde entier, et il constitue un problème majeur de santé publique. De toutes les plantes magiques, c'est sans conteste la mandragore qui, dès l'antiquité apparut comme la plus fabuleuse et la plus mystérieuse. Les plantes hallucinogènes, principalement présentes en Amérique, ont aussi une longue histoire. On a découvert des graines dans des sites paléolithiques. Très tôt, la pharmacopée sumérienne est riche de 250 plantes ou produits végétaux, dont la jusquiame. En 1550 av. J.C., la jusquiame et la mandragore sont mentionnées sur le papyrus Ebers de l'Égypte pharaonique, où elles voisinent avec l'opium et le chanvre. Dès cette époque, la jusquiame eșt connue et utilisée comme un poison violent. Un peu plus tard, en Grèce, certaines découvertes thérapeutiques sont attribuées aux héros et aux dieux ; à Hercule, l'héraclion ou Hyoscyamus albus. En effet, atteint de troubles nerveux, Hercule utilisait les effets sédatifs de cette plante. Dans l'ancien monde, datura metel constituait une plante sacrée associée aux rituels religieux de la Chine et de l'Inde, et était également connue dans la Grèce antique. En Grèce à partir du $8^{\mathrm{e}}$ siècle av. J.C., l'art de guérir, de magique, devient religieux. Dans «l'Odyssée» la belle Hélène verse le népenthès, un philtre, dans la coupe de vin de son époux «une substance qui dissipe la tristesse, calme la colère et fait oublier tous les maux». Il s'agit d'un mélange de datu- 
ra, d'opium et de chanvre. Hippocrate (460-377 av. J.C.) préconise l'usage de la mandragore par voie interne en alcoolat comme antidépresseur, antispasmodique et sédatif, ainsi que par voie externe pour les injections vaginales ou dans le traitement des hémorroïdes. Le «Corpus Hippocraticum» comprend plus de 230 drogues dont la mandragore, la jusquiame, la belladone. Galien (131-201) inventa la thériaque, médicament comportant 60 ingrédients, elle ne fut supprimée de la pharmacopée française qu'en 1908. En Gaule, dès l'époque romaine, on utilise la jusquiame pour ses propriétés antinévralgiques. Apulée au $4^{i}$ siècle, rédige «De virtutibus herbarum» dans lequel il précise «si l'on doit couper ou cautériser quelque membre, ou y porter le fer, que le patient boive une demi once de mandragore dans du vin, et il dormira jusqu'à ce que le membre soit coupé, sans éprouver aucune douleur». D'après De Gubernatis, la mandragore est la plante magique par excellence, c'est la plante des sorcières, à tel point que dans le pays des Goths, le mot alruna désigne à la fois la sorcière et la mandragore. Au moyen âge apparaissent les éponges soporifiques. Saint Benoît, au Mont Cassin décrit en 880 , l'inhalation à usage somnifère et antalgique d'un mélange de mandragore, jusquiame et opium. A cette époque, la mandragore était perçue comme la plante des sorcières. Selon la légende, la plante poussait un cri effroyable quand on voulait l'arracher et ceux qui tentaient de s'en emparer étaient foudroyés. Elle entrait dans la composition des philtres magiques. La mandragore est aussi connue pour être la plante qui naît de la terre fécondée par le sperme des pendus innocents. On lui prête pendant cette période des propriétés surnaturelles effrayantes. La jusquiame était utilisée au même titre que les solanacées vireuses (datura, mandragore), par les sorcières du moyen âge pour préparer les beuvrages et les pommades qui les emmenaient au sabbat en raison de leurs propriétés hallucinogènes. Les effets hallucinogènes de la jusquiame sont décrits par Avicenne (980-1037). En Amérique avant l'arrivée des espagnols, Datura ferox était déjà utilisée comme plante médicinale et comme hallucinogène. Les légendes concernant la mandragore sont nombreuses. L'une d'elles concerne Jeanne d'Arc qui fut soupçonnée d'en avoir possédé une comme l'évoque l'acte d'accusation dressé contre elle en 1430 : «Ladite Jeanne eut coutume de porter parfois une mandragore dans son sein, espérant par ce moyen avoir bonne fortune en richesse et choses temporelles». Une tradition veut toutefois que des mandragores poussassent à Domrémy, près de l'arbre sous lequel elle aurait entendu des voix. Dans le Pents-ts'ao (1505), la pharmacopée chinoise complétée au $16^{\mathrm{e}}$ siècle, le datura figure en bonne place parmi les substances végétales qui sont largement utilisées. En
1513, Machiavel a appelé une de ses comédies Mandragora, pièce dont le prétexte est l'utilisation du jus de mandragore comme fécondant des femmes stériles. Un autre mythe allemand fait de la mandragore, l'arbre du paradis terrestre. Avant Ambroise Paré (1510-1590), le chirurgien bolognais Théodoric pratiquait déjà le recours à l'anesthésie (1267). Ce dernier se servait d'éponges imprégnées de décoction d'opium, de jusquiame, d'huile de mandragore et de chanvre indien qu'il faisant inhaler au malade avant de l'opérer. Plus tard, à la renaissance, les alcaloïdes de la mandragore sont utilisés comme anesthésiques par Ambroise Paré. La poudre de datura a également été employée au $18^{\mathrm{e}}$ siècle par une bande de bandits parisiens appelés les endormeurs : ils offraient à leur future victime une prise de tabac mélangée à de la poudre de datura et profitaient de l'inconscience et de l'amnésie antérograde qui en résultait pour les détrousser. Le même type de scénario est mis en oeuvre en Colombie depuis les années 1970. Des bandes de délinquants extraient les alcaloïdes de Brugmansia arborea surnommé par les

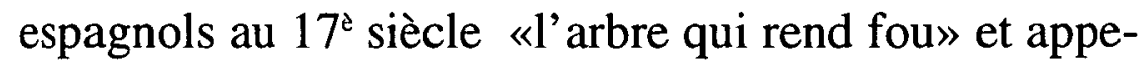
lé aujourd'hui «borrachero» (qui enivre), et les administrent à des personnes dans une boisson ou dans la nourriture, dans le but de les détrousser (la scopolamine fait perdre la volonté et est responsable d'une amnésie antérograde). La soumission de la victime permet aux délinquants de la dépouiller en toute tranquillité, tandis que l'amnésie leur assure l'impunité. C'est également pour cette raison que la scopolamine a été utilisée comme «sérum de vérité» pendant la seconde guerre mondiale.

\section{Formulaire magique :}

«On récole les mandragores, la nuit du vendredi, lorsqu'elles sont lumineuses après l'orage. Il convient en premier lieu, de tracer trois cercles concentriques autours de la plante, avec un poignard magique, puis ensuite de dégager délicatement la racine. Un chien noir affamé, animal condamné, est attaché au pied de la plante et, excité par le son du cor, est appelé au loin, devant franchir les trois cercles concentriques inscrits à terre. La plante émet lors de l'arrachage, un cri d'agonie insoutenable, tuant l'animal et l'homme non éloigné aux oreilles non bouchées de cire. La racine devient magique après lavage, macération et maturation en linceul ; elle représente l'ébauche de l'homme, "petit homme planté" ou "homonculus". Ainsi choyée, elle reste éternellement fidèle à son maître et procure à son possesseur, prospérité prodigieuse, abondance de biens et fécondité. De ce fait, elle est vendue très chère en raison du risque à la cueillette, de préférence sexuée par la présence de touffes judicieusement disposées. Une autre tradition concernant les alunes nous dit que la mandragore de forme humaine peut être considérée 
comme un esprit servant. Elle est indéfectiblement liée à celui qui l'a créé et ne peut être transmise qu'à sa famille qui en a la charge ou encore cédée à prix d'or. Celui qui achète une mandragore ne peut s'en débarrasser sauf à la vendre plus cher qu'il ne l'a achetée».

\section{Pharmacologie, pharmaco- cinétique, études contrô- lées, expérimentations ani- males (11)}

\section{- Le syndrome anticholinergique ou atro- pinique : aspects cliniques généraux :}

Le syndrome anticholinergique est produit par des substances de type atropine qui inhibent les effets de la stimulation de la fibre post ganglionnaire du parasympathique et possèdent à doses élevées une action excitatrice centrale. Elles s'opposent donc, par un blocage compétitif et réversible des récepteurs périphériques et centraux, à l'action de l'acétylcholine. Elles inhibent l'effet bradycardisant de celle-ci, ont une action antispasmodique au niveau des fibres musculaires lisses du tube digestif, des voies biliaires et urinaires, ralentissant la vidange gastrique et diminuant les sécrétions digestives, lacrymales et sudorales. Les signes et symptômes du syndrome anticholinergique qui sont liés à un blocage périphérique sont mydriase, sécheresse de la peau et des muqueuses, vasodilatation, hyperthermie, rétention urinaire, iléus et tachycardie. Les signes de blocage central sont confusion, agitation, hallucinations, myoclonies, tremblements, convulsions, coma et dépression respiratoire. La gravité du tableau clinique est très variable, dépendant de la nature du toxique en cause et de la quantité ingérée. Les formes bénignes sont les plus fréquentes, se limitant à une mydriase, une sécheresse buccale et une tachycardie sinusale, alors que les formes graves sont déterminées par la présence de signes neurologiques parfois dangereux sur le plan vital (Tableau I). Les toxiques le plus souvent en cause sont des médicaments (antidépresseurs tricycliques, certains antihistaminiques, antiparkinsoniens, antipsychotiques, antispasmodiques, myorelaxants, mais aussi préparations ophtalmiques). Les alcaloïdes des solanacées (belladone, datura, jusquiame en particulier) peuvent provoquer des intoxications sévères avec signes cardiaques et neurologiques. Le diagnostic est dans l'ensemble facile à évoquer; les signes ont parfois une bonne valeur d'orientation étiologique. Le traitement est symptomatique : hydratation, sédation, prévention ou traitement des convulsions, mesures de réanimation dans les cas les plus graves. La physostigmine, inhibi-
Tableau I : Atropine : relation effets-dose selon Goodman et Gilman (11).

\begin{tabular}{|c|l|}
\hline Dose & \multicolumn{1}{|c|}{ Effets } \\
\hline $0,5 \mathrm{mg}$ & $\begin{array}{l}\text { Légère diminution du rythme cardiaque, sécheresse de la bouche, } \\
\text { inhibition de la sudation }\end{array}$ \\
\hline $1,0 \mathrm{mg}$ & $\begin{array}{l}\text { Sécheresse buccale importante, soif, tachycardie, légère dilatation } \\
\text { des pupilles }\end{array}$ \\
\hline $2,0 \mathrm{mg}$ & $\begin{array}{l}\text { Rythme cardiaque rapide, palpitations, très forte sécheresse buc- } \\
\text { cale, dilatation des pupilles, vision proche floue }\end{array}$ \\
\hline $5,0 \mathrm{mg}$ & $\begin{array}{l}\text { Aggravation des symptômes, troubles d'élocution et de salivation, } \\
\text { lassitude et fatigue, céphalées, peau sèche et chaude, rétention } \\
\text { urinaire, diminution du péristaltisme intestinal }\end{array}$ \\
\hline $10,0 \mathrm{mg}$ & $\begin{array}{l}\text { Aggravation des signes cliniques, pouls rapide et filant, dilatation } \\
\text { pratiquement totale des pupilles, vision très floue, flush cutané, } \\
\text { peau chaude sèche et écarlate, ataxie, lassitude et excitation, } \\
\text { hallucinations et délire, coma }\end{array}$ \\
\hline
\end{tabular}

teur réversible des cholinestérases, a un effet sur l'encéphalopathie et la tachycardie mais son action est fugace et son usage controversé en raison d'effets secondaires parfois dangereux. Elle peut être recommandée avec prudence dans certaines formes graves : agitation extrême, coma convulsif, tachycardie supra ventriculaire sévère, mais elle n'est pratiquement pas utilisée en France.

\section{- Propriétés pharmacologiques :}

Bien que les structures chimiques soient très voisines, l'atropine et la scopolamine ont, à posologie identique, des propriétés pharmacologiques différentes. Ainsi, l'atropine -aux doses où elle est habituellement utilisée en thérapeutique- n'exerce que peu d'effets sur le système nerveux central. A l'inverse, la scopolamine développe essentiellement une action centrale aux doses thérapeutiques. Cette différence s'explique vraisemblablement par une plus grande perméabilité de la scopolamine à travers la barrière hémato-encéphalique.

\section{Action sur le système nerveux central :}

L'atropine, à doses thérapeutiques $(0,5$ à $1,0 \mathrm{mg})$ est responsable d'une stimulation vagale modérée. L'emploi de doses toxiques d'atropine produit une stimulation centrale avec agitation, irritabilité, désorientation, hallucinations ou délire. L'emploi de doses plus élevées conduit après une phase de stimulation, à une phase de paralysie et de coma puis à une dépression avec défaillance circulatoire, et dépression respiratoire. A l'inverse, la scopolamine à doses thérapeutiques est responsable d'une dépression avec somnolence, amnésie, fatigue. Elle est également susceptible de causer une certaine euphorie à l'origine d'abus. A plus fortes doses, lẹs effets sont voisins de ceux constatés avec des doses toxiques d'atropine, à type de stimulation centrale avec agitation, hallucinations ou délire. L'atropine est davantage prescrite en raison de ses effets beaucoup plus limi- 
tés sur le système nerveux central que la scopolamine.

Action sur le système nerveux autonome :

$\mathrm{Au}$ niveau du système nerveux autonome, la neurotransmission cholinergique s'effectue par l'intermédiaire des récepteurs cholinergiques nicotiniques. L'atropine exerce un effet inhibiteur sur les récepteurs muscariniques périphériques par antagonisme compétitif de l'acétylcholine.

\section{Action oculaire :}

Les antagonistes du récepteur muscarinique empêchent la stimulation cholinergique du muscle sphinctérien de l'iris et du muscle ciliaire du cristallin aboutissant à une dilatation de la pupille (mydriase) et à une paralysie de l'accommodation (cycloplégie). Cependant, une dose de $0,6 \mathrm{mg}$ d'atropine par voie générale n'exerce qu'un faible effet oculaire alors la même dose de scopolamine donne une mydriase et une cycloplégie marquées.

\section{Action cardiovasculaire :}

- Action sur le coeur : bien que l'effet majeur constaté est la tachycardie, à doses thérapeutiques $(0,4$ à $0,6 \mathrm{mg}$ pour l'atropine - 0,1 à $0,2 \mathrm{mg}$ pour la scopolamine) une bradycardie temporaire est observée, celle-ci est plus marquée avec la scopolamine. Des doses plus importantes d'atropine sont responsables d'une tachycardie, 35 à 40 pulsations supplémentaires par minute pour $2 \mathrm{mg}$ par voie IM. Avec la scopolamine, on ne constate pas de tachycardie à la posologie ayant une action oculaire, contrairement à l'atropine.

- Action sur la circulation : l'atropine, à doses thérapeutiques, n'exerce pas d'effet significatif, à l'exception de la neutralisation de la vasodilatation périphérique et de la chute de la pression artérielle produites par les esters de la choline. L'atropine à doses toxiques, ou même parfois thérapeutiques, dilate les vaisseaux cutanés, en particulier ceux du visage donnant un aspect rouge caractéristique (flush atropinique).

\section{Action sur l'appareil respiratoire :}

Les alcaloïdes de la belladone inhibent les sécrétions au niveau du nez, de la bouche, du pharynx, des bronches et de ce fait sèchent toutes les muqueuses de l'appareil respiratoire. Cette propriété est à la base de l'emploi de l'atropine et de la scopolamine en médication préanesthésique. On note également un effet broncho-dilatateur.

\section{Action sur le tractus gastro-intestinal :}

Les alcaloïdes exercent essentiellement une action antispasmodique, mais ils inhibent aussi la sécrétion salivaire et gastrique, et partiellement la sécrétion intestinale. Ils diminuent la motilité gastro-intestinale.

\section{Action sur les autres muscles lisses :}

Les effets les plus marqués concernent les voies urinaires susceptibles de se dilater sous l'effet de l'atropine.

\section{- Pharmacocinétique :}

Les alcaloïdes hallucinogènes de ces solanacées sont rapidement absorbés à partir du tube digestif, ils exercent de ce fait très vite leurs effets pharmacologiques. Leur métabolisme est essentiellement hépatique et contribue pour $50 \%$ à leur élimination, la fraction non métabolisée étant éliminée sous forme inchangée dans les urines. Les principales caractéristiques de la pharmacocinétique des alcaloïdes de ces solanées sont regroupées Tableau II.

Tableau II : Principales caractéristiques pharmacocinétiques des alcaloüdes des solanées hallucinogènes $(12,13)$.

\begin{tabular}{|l|c|c|}
\hline & $\begin{array}{c}\text { Atropine } \\
\text { (DL-hyoscyamine) }\end{array}$ & $\begin{array}{c}\text { Scopolamine } \\
\text { (L-hyoscine) }\end{array}$ \\
\hline Demi-vie (heure) & $2-5$ & $3-8$ \\
\hline Volume de distribution $(\mathrm{l} / \mathrm{kg})$ & $1-6$ & 1,4 \\
\hline Concentrations thérapeutiques $(\mathrm{ng} / \mathrm{ml})$ & $2-25$ & $0,1-1,0$ \\
\hline Concentrations toxiques $(\mathrm{ng} / \mathrm{ml})$ & $20-30$ & - \\
\hline Concentrations létales $(\mathrm{ng} / \mathrm{ml})$ & $>200$ & - \\
\hline
\end{tabular}

\section{Effets aigus et chroniques chez l'homme $(4,14)$}

\section{- Effets aigus :}

Ces plantes solanées appartiennent dans la classification de Lewin au $2^{\text {eme }}$ groupe «Phantastica» des agents hallucinants. En cas d'exposition aiguë, outre un état d'agitation intense induit par les hallucinations et les convulsions, on note des troubles cardiaques, un coma, une dépression respiratoire. La présence de signes neurologiques doit attirer l'attention, car ils présentent un danger sur le plan vital. La gravité du tableau clinique est très variable et est fonction de la quantité ingérée. Une confusion mentale, des hallucinations, suivis d'un coma convulsif associé à une mydriase doivent faire évoquer un syndrome anticholinergique grave. Pour la belladone, chez l'enfant, on admet que la dose toxique -voire létale- se situe au delà de 2 baies $(1 \mathrm{à} 2 \mathrm{mg}$ d'atropine dans une baie ; le décès étant possible pour des doses de $0,2 \mathrm{mg} / \mathrm{kg}$ ). Chez l'adulte, la consommation de plus de 10 baies conduit à une intoxication grave et justifie l'hospitalisation. Pour l'atropine, la dose létale chez l'adulte est supérieure à $10 \mathrm{mg}$. Il convient d'indiquer également la forte sensibilité individuelle à l'atropine. Pour le datura, plus riche en scopolamine, la dose toxique chez l'enfant est de 2 à $5 \mathrm{~g}$ de graines $(0,1 \mathrm{mg} / \mathrm{kg}$ exprimé en scopolamine). La dose létale chez l'adulte est de 10 à $12 \mathrm{~g}$ de graines (soit plus de 2 à $4 \mathrm{mg}$ de scopolamine). 


\section{- Effets chroniques :}

En ce qui concerne le phénomène d'addiction, pour les alcaloïdes de ces solanacées hallucinogènes, contrairement à de nombreuses autres drogues, peu de phénomènes de dépendance psychique ou physique, ni de tolérance sont rapportés. Les complications psychiatriques en revanche sont plus fréquentes, bouffées délirantes, ou révélation d'une psychose schizophrénique.

\section{Utilisation en thérapeu- tique (15-19)}

\section{- Extraits et teintures végétaux :}

Deux extraits figurent à la pharmacopée française :

- l'extrait ferme titré de feuille de belladone (2000),

- l'extrait de jusquiame noire (1989).

«L'extrait ferme titré de feuille de belladone est obtenu à partir de feuille de belladone. Il contient au minimum $2,3 \%$ et au maximum $2,7 \%$ d'alcaloïdes totaux exprimés en hyoscyamine, calculés par rapport à un extrait à $90 \%$ de résidu sec».

Deux teintures sont également inscrites au codex :

- la teinture de belladone (1996),

- la teinture de jusquiame noire (1989).

De très nombreuses spécialités contenant des extraits végétaux de belladone, de datura, de jusquiame ou de mandragore ont été commercialisées par le passé ; il ne reste plus aujourd'hui essentiellement que quelques spécialités homéopathiques. Parmi les spécialités allopathiques contenant de la poudre de belladone, en association avec d'autres principes actifs citons les gélules de Gélumaline ${ }^{\circledR}$ et les suppositoires de Suppomaline ${ }^{\circledR}$.

\section{- Alcaloïdes utilisés en thérapeutique :}

Atropine :

Vingt huit spécialités contenant de l'atropine sont encore commercialisées. Il s'agit essentiellement d'ampoules injectables $(0,25-0,5$ et $1 \mathrm{mg} / 1 \mathrm{ml})$ ou de collyres $(0,3-0,5$ ou $1 \%)$. Les indications sont celles de l'atropine par voie injectable (SC ou IV) ou des collyres. Parmi ces vingt huit médicaments, deux ont pour principe actif l'atropine oxyde (Génatropine ${ }^{\circledR}$, comprimés et solution buvable), utilisé dans le traitement symptomatique des douleurs liées aux troubles fonctionnels du tube digestif et des voies biliaires. En association, l'atropine est employée dans le traitement symptomatique de la diarrhée chez l'adulte et chez l'enfant (Diarsed $\circledast$ ).

\section{Hyoscyamine:}

Une seule spécialité figure au dictionnaire Vidal, le Sulfate de Duboisine ${ }^{\circledR}$, collyre utilisé chez les sujets allergiques à l'atropine.

\section{Scopolamine :}

Cinq spécialités contenant de la scopolamine sont commercialisées en France, quatre sous forme de bromhydrate :

- Scopolamine ${ }^{\circledR}$ (Cooper ou Pharmacie Centrale des Hôpitaux) en solution injectable pour ses propriétés anticholinergiques, antiémétiques, antisécrétoires salivaires et antispasmodiques.

- Scopoderm TTS® en dispositif transdermique délivrant $1 \mathrm{mg}$ en 72 heures dont l'indication thérapeutique principale est le mal des transports.

La cinquième spécialité, sous forme de N-butyl bromure (Scoburen $\circledast$ ), antispasmodique et anticholinergique est essentiellement utilisée dans les manifestations douloureuses aiguës en gynécologie ainsi que dans les occlusions intestinales. Enfin le chlorhydrate et le bromhydrate aminoxyde de scopolamine ne sont pas commercialisés en France.

\section{Identification et dosage $(6,15,20-26)$}

- Dans les végétaux :

Dans la pharmacopée française, l'extrait de feuilie de belladone préparé par lixiviation de feuilles de belladone divisées dans l'éthanol à $70 \%(\mathrm{v} / \mathrm{v})$ doit satisfaire un certain nombre d'essais. Il s'agit de l'identification des alcaloïdes par CCM après extraction en phase liquide puis révélation colorimétrique, et dosage volumétrique de la teneur en alcaloïdes totaux exprimés en hyoscyamine. Des méthodes de recherche multirésidus dans les végétaux ont été mises au point. La technique de Hostege en particulier fait appel à une double extraction en phase liquide suivie d'une purification en phase solide. L'analyse quantitative est effectuée par CG-NPD et l'analyse qualitative par CG-SM et CCM. Les rendements d'extraction sont compris entre 72 et $113 \%$ à la concentration de $1 \mu \mathrm{g} / \mathrm{g}$.

- Dans les milieux biologiques :

L'analyse toxicologique des alcaloïdes de ces solanacées est extrêmement délicate en raison des faibles concentrations susceptibles d'exercer une action pharmacologique. Ainsi pour l'atropine, la CG-SM après dérivation puis acquisition en mode sélection d'ions, la chromatographie liquide avec paire d'ions, ou l'électrophorèse capillaire, présentent des limites de quantification respectives de $10 \mathrm{ng} / \mathrm{ml}, 400 \mathrm{ng} / \mathrm{ml}$ et 200 $\mathrm{ng} / \mathrm{ml}$, ce qui est insuffisant pour réaliser des dosages sanguins. La CG-SM et la CL-BD sont cependant utiles pour mettre en évidence les principes actifs lorsque ceux ci sont présents en grande quantité dans les urines 
ou le contenu gastrique. La technique par CL-SM proposée par Gaillard (6) et Cheze (24) répond bien en revanche aux critères de sensibilité pour le dosage sanguin des alcaloïdes majeurs ; pour l'atropine aussi bien en suivi thérapeutique que dans un cadre toxicologique, et pour la scopolamine en cas d'intoxication (Tableau III). Les principes actifs sont extraits par une triple extraction en phase liquide, l'extrait final est repris par l'acétonitrile. La séparation chromatographie est réalisée sur colonne C18-ODS avec un gradient de phase (tampon/acétonitrile/méthanol). La détection est effectuée en mode positif. La figure 2 montre le chromatogramme d'un extrait de sang total dans un cas d'intoxication par datura. L'emploi de la CL-SM-SM permet outre d'améliorer les performances de la technique décrite avec le gain d'un facteur voisin de 10 pour la limite de détection, ce qui permet de quantifier la scopolamine dans le sang en suivi thérapeutique. Xu et coll. proposent une technique de dosage de la L-hyoscyamine par CL-SM-SM avec une limite de quantification de $0,02 \mathrm{ng} / \mathrm{ml}$. Oertel et coll. en utilisant la même technique, pour la scopolamine, après une extraction en phase solide obtiennent une limite de quantification de $0,02 \mathrm{ng} / \mathrm{ml}$.

Tableau III : Étude de la reproductibilité quotidienne, rendement et limite de détection (LD) des alcaloüdes par CL-SM selon Gaillard et Cheze $(6,24)$.

\begin{tabular}{|c|c|c|c|c|c|c|}
\hline \multirow{2}{*}{ Composé } & \multicolumn{4}{|c|}{ Coefficient de variation $(\%)$ à } & \multirow{2}{*}{$\begin{array}{c}\text { Rendement } \\
\text { d'extraction } \\
(\%)\end{array}$} & \multirow{2}{*}{$\begin{array}{r}\text { LD } \\
(\mathrm{ng} / \mathrm{ml}) \\
(\mathrm{n}=30)\end{array}$} \\
\hline & $\begin{array}{c}0,1 \\
\mathrm{ng} / \mathrm{ml}\end{array}$ & $\begin{array}{c}1 \\
\mathrm{ng} / \mathrm{ml}\end{array}$ & $\begin{array}{c}10 \\
\mathrm{ng} / \mathrm{ml}\end{array}$ & $\begin{array}{r}100 \\
\mathrm{ng} / \mathrm{ml}\end{array}$ & & \\
\hline Atropine & 11,8 & 6,1 & 4,2 & 3,7 & 70,9 & 0,09 \\
\hline Scopolamine & - & 12,4 & 9,1 & 6,9 & 64,4 & 0,31 \\
\hline
\end{tabular}

\section{Observations médicales et médico-légales $(14,20,27-30)$}

- Intoxications par les alcaloïdes des solanacées : le datura est le plus prisé sous nos latitudes :

L'intoxication collective involontaire la plus connue par les solanacées concerne la belladone. Le $1^{\text {er }}$ septembre 1825 , elle atteignit 160 soldats du $12^{\mathrm{e}}$ régiment d'infanterie. Assoiffés par une longue marche, ils avaient découvert au cours d'une pause des plantes chargées de fruits qui ressemblaient tellement à des guignes que la plupart des soldats en consommèrent. Bientôt les premiers signes apparaissent : soif intense, nausées, vomissements, tachycardie. Certains soldats manifestent des signes d'atteinte psychique, se mettent à chanter, à danser ; d'autres sont pris de terreur, hallu- cinés ou présentent des convulsions. Plusieurs présentent une rougeur caractéristique de la face ou du cou. Tous ont des troubles visuels et une mydriase.

Pour la belladone, les observations médicales sont rares, il s'agit le plus souvent d'intoxications volontaires. Schneider et coll. ont publié huit cas, dont trois ont présenté des hallucinations visuelles. A l'inverse de la belladone, en ce qui concerne le datura, les observations sont nombreuses. Ainsi les publications des trois dernières années font essentiellement état d'intoxications volontaires chez des sujets jeunes à la recherche des effets hallucinogènes. Au cours de cette période, on note plusieurs dizaines de cas, localisés dans le sud de la France avec semble-t-il une forte progression. Djibo et coll. (27) rapportent quatre cas d'intoxication au Niger où les fleurs et les graines de datura sont utilisées pour leurs propriétés hallucinogènes. Les quatre malades avaient fumé des fleurs séchées de la plante et consommé une infusion de thé contenant des graines de datura. La symptomatologie d'évolution favorable est dominée par des signes neurologiques en particulier, une désorientation temporo-spatiale, une agitation et des hallucinations polymorphes chez les quatre sujets associés à une mydriase, une fièvre, une tachycardie, une soif, une sécheresse de la bouche. En 2002, Birmes et coll. (28) décrivent, dans région de Toulouse, trois cas d'intoxication volontaire par datura avec syndrome anticholinergique central typique : agitation, confusion, hallucinations et comportements agressifs. Le pronostic fut bon pour les trois sujets mais une hospitalisation a été nécessaire en raison de la sévérité des troubles psychiatriques. En 2003, Arouko et coll. (14), dans le bordelais, constatent une recrudescence récente de l'utilisation de plantes hallucinogènes au cours des soirées festives. Datura stramonium est comme plébiscité ces derniers temps et les hospitalisations pour intoxications aiguës par cette plante se multiplient comme l'indiquent les chiffres du Centre Antipoison de Bordeaux (14 hospitalisations pour intoxication volontaire par le datura en 2002 contre 5 en 2001). Arouko et coll. soulignent que même si l'évolution est favorable, les retards de diagnostic posent des problèmes car le médecin consulté en urgence n'y pense pas. Bernard du Centre d'Évaluation et d'Information sur la Pharmacodépendance de Lyon rapporte au cours de l'été 2003 cinq cas d'intoxications volontaires au datura chez des jeunes de 16 à 19 ans. Tous ont été hospitalisés en raison d'hallucinations principalement visuelles et parfois auditives, associées à une agitation et à une mydriase. La guérison intervient dans tous les cas en quelques heures à quatre jours. 

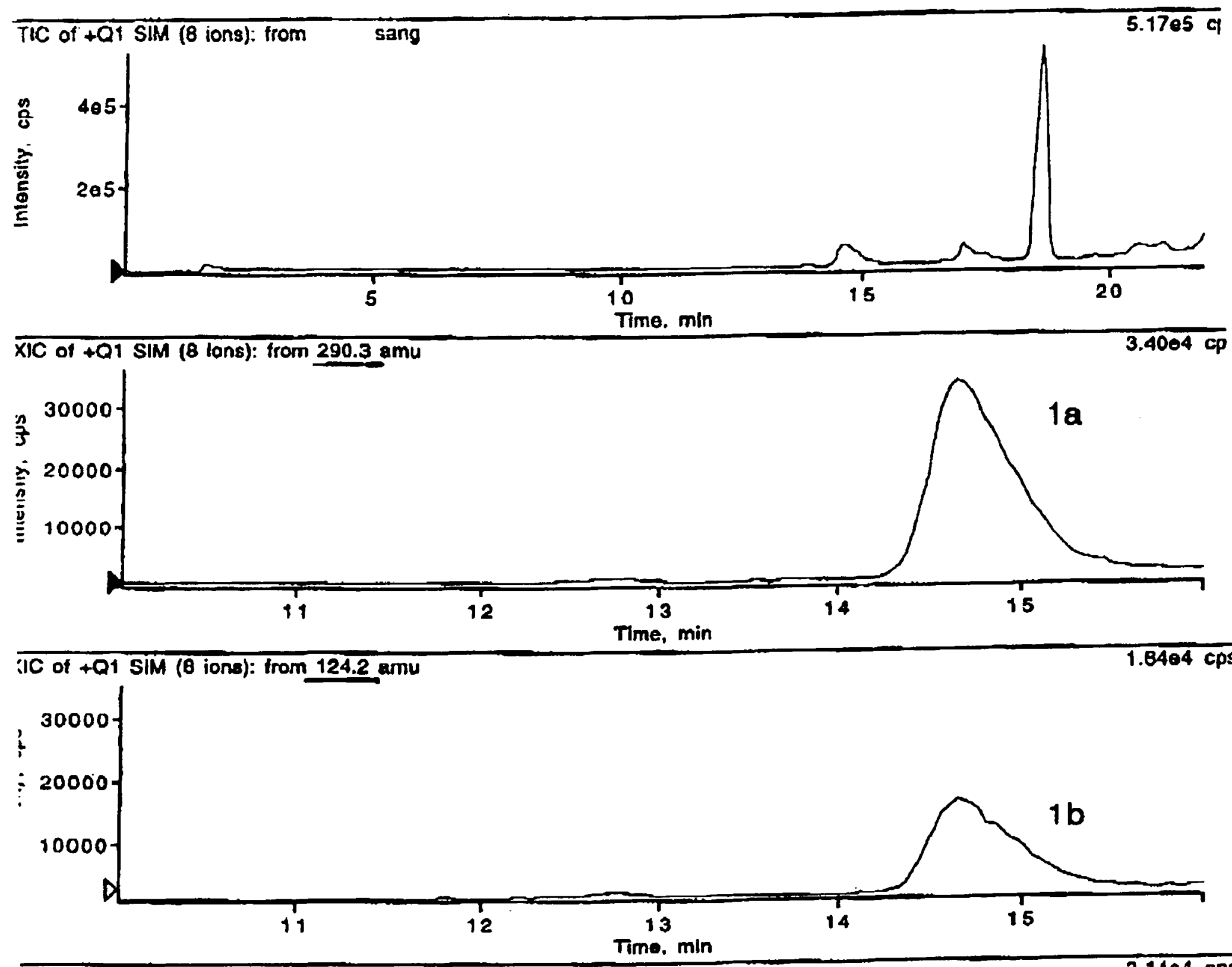

IC of to. SiM (8 ions): from 304.3 amu
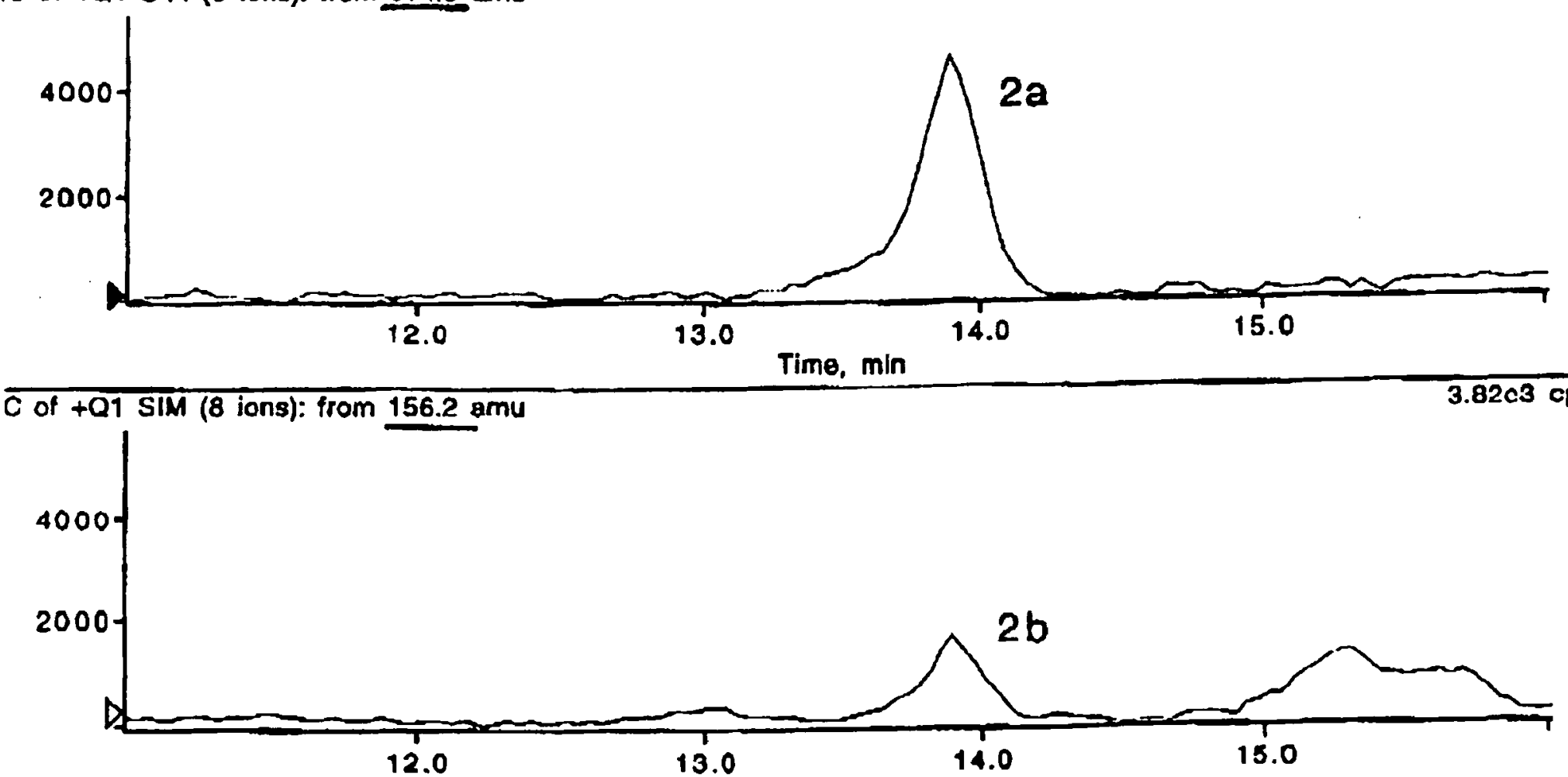

Figure 2 : Chromatogramme sur colonne ODS d'un extrait de sang total. Pics la et $16=$ atropine $(\mathrm{m} / 2$ 290,3 et 124,2), 2a et $2 b$ = scopolamine $(\mathrm{m} / \mathrm{z} 304,3$ et 156,2$)$. 


\section{- Observations médicales et médico- légales personnelles :}

\section{Cas $n^{\circ} 1$ et $n^{\circ} 2$ :}

Deux jeunes soeurs jumelles âgées de 7 ans et souffrant de problèmes psychomoteurs depuis leur enfance sont retrouvées brûlées dans leur habitation, comme endormies, sans signe de tentative de fuite. L'enquête a finalement déterminé l'incendie comme étant d'origine criminelle et a permis de trouver au domicile familial des ouvrages sur les plantes toxiques. L'autopsie montrait des signes de violences ainsi que des résidus de fumées dans les trachées. Des prélèvements de sang, de cheveux et de contenu gastrique étaient effectués. Une analyse de première intention révélait la présence de toxiques dus à l'incendie ( $\mathrm{HbCO}$, cyanures) et à un traitement anti-épileptique (acide valproïque, clonazépam, carbamazépine) qui n'expliquaient pas à eux seuls l'absence de réaction des fillettes face au feu. Des analyses complémentaires étaient donc réalisées pour la recherche des toxiques végétaux (Tableau IV). Le screening des alcaloïdes révélait la présence d'atropine et de scopolamine dans le sang et le contenu gastrique à des concentrations non mortelles, cependant l'effet narcotique du datura (hallucinogène narcotique incapacitant) les avait probablement laissées dans un état de stupeur semi-comateux pouvant expliquer leur incapacité à fuir. Les parents ont finalement avoué avoir drogué leurs enfants avec des extraits de feuilles de datura, fréquente dans cette région, puis les avoir tuées par le feu après que leur requête d'admission de celles-ci dans une institution spécialisée fut refusée.

\section{Cas $n^{\circ} 3$ et 4 :}

Loïc, 15 ans et Jean-Paul, 16 ans, sont hospitalisés après une fête techno-rave à Biscarosse. Des échantillons de capsules et graines de datura qu'ils sont susceptibles d'avoir consommé sont saisis. A l'admission, ils présentent tous deux un syndrome atropinique marqué avec tachycardie et hallucinations. Les prélèvements sanguins, réalisés plusieurs heures après l'hospitalisation, ne permettent pas de mettre en évidence l'atropine et la scopolamine. On note en revanche la présence de cannabis.

\section{Cas $n^{\circ} 5$ :}

Karl, 33 ans, disparaît à la sortie d'une discothèque Bordelaise. Son corps est retrouvé 10 jours plus tard flottant dans la Garonne. Les constatations autopsiques sont celles d'un décès par noyade. L'expertise toxicologique révèle dans le sang la présence d'éthanol $(1,38$ $\mathrm{g} / \mathrm{l}$ ), de THC et de THC-COOH (4 et $6,8 \mathrm{ng} / \mathrm{ml}$ ), mais l'absence d'atropine et de scopolamine. Les urines montrent la présence de THC-COOH $(79 \mathrm{ng} / \mathrm{ml})$, ainsi que de l'atropine et de la scopolamine. Lors d'intoxi- cations par datura, les décès sont rarement liés à une overdose d'alcaloïdes. Ceux-ci sont le plus souvent la conséquence d'actes inconsidérés dus aux troubles psychiques engendrés essentiellement par la scopolamine. En effet, risque suicidaire et inconscience totale du danger sont responsables de défenestrations et de noyades.

\section{Cas n ${ }^{\circ}$ :}

Christophe, 28 ans, connu pour une toxicomanie ancienne est retrouvé mort à son domicile en Charente. Le décès remontre à plus d'une semaine. Dans la poche du vêtement qu'il porte, on retrouve des graines de datura. Les constatations autopsiques font état d'une congestion multiviscérale et d'une putréfaction importante du corps. Le liquide hématique prélevé montre la présence de loxapine, de morphine et d'atropine. Les urines révèlent la présence d'atropine et de scopolamine. De l'atropine est également décelée dans la bile.

\section{Conclusion}

En raison de leurs propriétés hallucinogènes, la consommation des diverses parties de ces solanacées et plus particulièrement de datura, qui semble être plébiscité à l'heure actuelle, est une cause d'intoxication. Celle-ci peut être à l'origine d'une hospitalisation, surtout chez des sujets jeunes à la recherche de sensations fortes. Les signes cliniques orientent le clinicien, mais le biologiste doit être en mesure de confirmer la présence des alcaloïdes dans les milieux biologiques. En médecine légale, il est important de rappeler que les troubles du comportement, induits par ces principes actifs, dont la scopolamine présente en grande quantité dans le datura, peuvent être à l'origine d'actes inconsidérés dus aux troubles psychiques et responsables de suicides. 
Tableau IV : Résultats de l'analyse toxicologique des cas $n^{\circ} 1$ et $n^{\circ} 2$.

\begin{tabular}{|l|c|l|c|}
\hline Fillette 1 & Fillette 2 \\
\hline Sang & Carboxyhémoglobine & Sang & \\
& (HbCO) $=11,3 \%$ & & HbCO $=11,9 \%$ \\
& Acide valpröque $=14,4 \mu \mathrm{g} / \mathrm{ml}$ & & Acide valpröque $=8,3 \mu \mathrm{g} / \mathrm{ml}$ \\
& Clonazépam $=0,9 \mu \mathrm{g} / \mathrm{ml}$ & Clonazépam $=1,9 \mu \mathrm{g} / \mathrm{ml}$ \\
& Cyanure $=2,4 \mu \mathrm{g} / \mathrm{ml}$ & & Cyanure $=0,7 \mu \mathrm{g} / \mathrm{ml}$ \\
& Atropine $=\mathbf{3 2 , 5} \mathbf{~ n g} / \mathbf{m l}$ & & Atropine $=\mathbf{7 , 5} \mathbf{~ n g} / \mathbf{m l}$ \\
& Scopolamine $=\mathbf{4 , 4} \mathbf{~ n g} / \mathbf{m l}$ & & Scopolamine $=\mathbf{0 , 8} \mathbf{~ n g} / \mathbf{m l}$ \\
\hline Contenu & Atropine $=\mathbf{2 2 , 0} \mathbf{~ n g / m l}$ & Contenu & Atropine $=\mathbf{1 8 , 3} \mathbf{~ n g} / \mathbf{m l}$ \\
Gastrique & Scopolamine $=\mathbf{2 , 1} \mathbf{~ n g} / \mathbf{m l}$ & Gastrique & Scopolamine $=\mathbf{1 , 1} \mathbf{~ n g} / \mathbf{m l}$ \\
\hline
\end{tabular}

\section{Références}

1. Goullé J.P., Droy J.M., Leroy J.P. Réponse analytique aux syndromes cholinergique et anticholinergique. Ann. Toxicol. Anal. $2000 ; 12: 282-95$.

2. Résultats de l'enquête ESCAPAD 2002. Observatoire français des drogues et des toxicomanies. www.ofdt.fr

3. Guignard J.L. Abrégé de botanique. Masson Ed. Paris, 1998.

4. www.toxibase.org

5. Fabre R., Truhaut R. Précis de toxicologie. Tome II. Sedes Ed. Paris, 1971.

6. Gaillard Y., Pepin G. Poisoning by plant material : review of human cases and analytical determination of main toxins by high-performance liquid chromatography-(tandem) mass spectrometry. J. Chromatogr. B. 1999 ; 733 : 181-229.

7. Schultes R.E., Hofmann A. Les plantes des dieux. Les plantes hallucinogènes, botanique et ethnologie. Ed. du Lézard. Paris, 1993.

8. Levy M.N., Schwartz P.J. Ed. Vagal control of the heart : experimental basis and clinical implications. Futura Publishing Co., Armonk, NY, 1994.

9. Symposium (divers auteurs). Subtypes of muscarinic receptors V. (Levine R.R., Birdsall N.J.M. Ed.). Life Sci. 1993 ; 52 : 405-597.

10. Pelt J.M. Drogues et plantes magiques. Fayard, Paris, 1996.

11. Brown J.H., Taylor P. Muscarinic receptor agonists and antagonists. The pharmacological basis of therapeutics. Hardman J.G., Limbird L.E. Ed. 1990 : 141-60.

12. Wennig R. Laboratory diagnosis of poisonings. Human Toxicology. Descotes J. Elsevier Ed. 1996 : 25-236.

13. Uges DRA. Therapeutic and toxic drug concentration. TIAFT Bulletin. 1996 ; 26 (1 suppl.) : 34 pp.

14. Arouko H., Matray M.D., Bragança C., Mpaka J.P., Chinello L., Castaing F., Bartou C., Poisot D. L'intoxication volontaire par ingestion de Datura stramonium. Une autre cause d'hospitalisation des jeunes en quête de sensations fortes. Ann. Med. Interne. 2003 ; 154 Hors série I : 1S46-1S50.

15. Arrêté du 5 juin 2000 portant additif n46 à la pharmacopée française (JO du 19 juillet 2000).

16. Pharmacopée française. Dixième édition (1982).

17. www.vidalpro.net
18. www.theriaque.org/InfoMedicaments/

19. www.biam2.org/www/Isub.html

20. Hostege D.M., Seiber J.N., Galey F.G. Rapid multiresidue screen for alkaloids in plant material and biological samples. J. Agric. Food Chem. 1995 ; 43 : 691-9.

21. Saady J.J., Poklis A. Determination of atropine in blood by gas chromatography/mass spectrometry. J. Anal. Toxicol. $1989 ; 13: 296-9$.

22. Buch U., Isenberg E., Buch H.P. HPLC assay for atropine in serum and protein solutions after in vitro addition of the tropane alkaloid. Methods Find. Exp. Clin. Pharmacol. 1994 ; $16: 361-5$.

23. Plaut O., Staub C. Determination of atropine in biological fluids by micellar electrokinetic capillary chromatography in the presence of strychnine and tetracaine. Electrophoresis. $1998 ; 19: 3003-7$.

24. Cheze M., Gaillard Y., Pépin G. Réponses analytiques aux intoxications par substances végétales. Ann. Toxicol. Anal. $2000 ; 12: 307-14$.

25. Xu A., Havel J., Linderholm K., Hulse J. Development and validation of an LC/MS/MS method for the determination of L-hyoscyamine in human plasma. J. Pharm. Biomed. Anal. $1995 ; 14$ : 33-42.

26. Oertel R., Richter K., Ebert U., Kirch W. Determination of scopolamine in human serum and microdialysis samples by liquid chromatography-tandem mass spectrometry. J. Chromatogr. B. Biomed. Sci. Appl. 2001 ; 750 : 121-8.

27. Djibo A., Brah Bouzou S. Intoxication aiguë au «Sobilobi» (Datura). A propos de quatre cas au Niger. Bull. Sco. Pathol. Exot. 2000 ; 93 : 294-7.

28. Birmes P., Chounet V., Mazerolles M., Cathala B., Schmitt L., Lauque D. Intoxication volontaire par Datura stramonium. Trois observations. Presse Med. 2002 ; 31 : 69-72.

29. Bernard N. Consommation de Datura : à propos de 5 hospitalisations notifiées au CEIP de Lyon au cours de l'été. VIGItox $2003 ; 22: 3$.

30. Schneider F., Luntun P., Kintz P., Astruc D., Flesch F., Tempe J.D. Plasma and urine concentrations of atropine after the ingestion of cooker deadly nightshade berries. J. Toxiol. Clin. Toxicol. $1996 ; 34$ : 113-7. 